\title{
Higher Enhancement Intrahepatic Nodules on the Hepatobiliary Phase of Gd-EOB-DTPA- Enhanced MRI as a Poor Responsive Marker of Anti-PD-1/PD-L1 Monotherapy for Unresectable Hepatocellular Carcinoma
}

\author{
Tomoko Aoki ${ }^{a}$ Naoshi Nishida ${ }^{a}$ Kazuomi Ueshima ${ }^{a}$ Masahiro Morita ${ }^{a}$ \\ Hirokazu Chishina ${ }^{a}$ Masahiro Takita ${ }^{a}$ Satoru Hagiwara ${ }^{a}$ Hiroshi Ida ${ }^{a}$ \\ Yasunori Minami $^{a}$ Akira Yamada $^{b}$ Keitaro Sofue ${ }^{c}$ Masakatsu Tsurusaki ${ }^{d}$ \\ Masatoshi Kudo ${ }^{\mathrm{a}}$
}

${ }^{a}$ Department of Gastroenterology and Hepatology, Kindai University Faculty of Medicine, Osaka-Sayama, Japan;

${ }^{b}$ Department of Radiology, Shinshu University School of Medicine, Nagano, Japan; 'Department of Radiology, Kobe University Graduate School of Medicine, Kobe, Japan; 'Department of Radiology, Kindai University Faculty of Medicine, Osaka-Sayama, Japan

\section{Keywords}

Anti-programmed cell death protein $1 \cdot$ Programmed death-ligand 1 antibody · Hepatocellular carcinoma . Gadolinium-ethoxybenzyl-diethylenetriamine-enhanced magnetic resonance imaging $\cdot \mathrm{Wnt} / \beta$-catenin $\cdot$ Imaging biomarker

\begin{abstract}
Introduction: Immune checkpoint inhibitors (ICls) are promising agents for the treatment of hepatocellular carcinoma (HCC). However, the establishment of noninvasive measure that could predict the response to $\mathrm{ICl}$ s is challenging. This study aimed to evaluate tumor responses to $\mathrm{ICls}$ using the hepatobiliary phase of gadolinium-ethoxybenzyl-diethylenetriamine (Gd-EOB-DTPA)-enhanced magnetic resonance imaging (MRI), which was shown to reflect Wnt/ $\beta$-catenin activating mutation. Methods: A total of 68 intrahepatic HCC nodules from 18 patients with unresectable HCC and ChildPugh class $A$ liver function who received anti-programmed
\end{abstract}

karger@karger.com www.karger.com/lic

Karger $\frac{1}{\%}$
(C) 2021 The Author(s)

Published by S. Karger AG, Basel

This is an Open Access article licensed under the Creative Commons Attribution-NonCommercial-4.0 International License (CC BY-NC) (http://www.karger.com/Services/OpenAccessLicense), applicable to the online version of the article only. Usage and distribution for commercial purposes requires written permission. cell death 1 (PD-1)/programmed death-ligand 1 (PD-L1) monotherapy were enrolled in this study. All patients had viable intrahepatic lesions evaluable using the hepatobiliary phase of Gd-EOB-DTPA-enhanced MRI within the 6 months prior to the treatment. The relative enhancement ratio was calculated, and the time to nodular progression (TTnP) defined as $20 \%$ or more increase in each nodule was compared between higher or hypo-enhancement HCC nodules. Then, the progression-free survival (PFS) and objective response rate (ORR) per Response Evaluation Criteria in Solid Tumors version 1.1 (RECIST v1.1) were compared between patients with and without HCC nodules with higher enhancement on hepatobiliary phase images. Results: The median PFS was 2.7 (95\% confidence interval [Cl]: 1.4-4.0) months in patients with HCC nodules with higher enhancement $(n=8)$ and 5.8 (95\% Cl: 0.0-18.9) months in patients with hypointense HCC nodules $(n=10)(p=0.007)$. The median TTnP of HCC nodules with higher enhancement $(n=23)$ was 1.97 (95\% Cl: $1.86-$ $2.07)$ months and that of hypointense HCC nodules $(n=45)$ was not reached $(p=0.003)$. The ORR was $12.5 \%(1 / 8)$ versus 
$30.0 \%(3 / 10)$; the disease control rate was $37.5 \%(3 / 8)$ versus $70.0 \%(7 / 10)$, respectively, in patients with or without higher enhancement intrahepatic HCC nodules. Conclusion: The $\mathrm{TnP}$ on HCC nodules with higher enhancement and the median PFS in patients who carried higher enhancement intrahepatic HCC nodules were significantly shorter than those in hypointense HCC nodules with anti-PD-1/PD-L1 monotherapy. The intensity of the nodule on the hepatobiliary phase of Gd-EOB-DTPA-enhanced MRI is a promising imaging biomarker for predicting unfavorable response with anti-PD-1/ PD-L1 monotherapy in patients with HCC.

(C) 2021 The Author(s).

Published by S. Karger AG, Basel

\section{Introduction}

Hepatocellular carcinoma (HCC) is one of the leading causes of cancer-related mortality worldwide, and many patients present with an unresectable disease [1-4]. In this scenario, systemic chemotherapy is required, which includes several molecular targeted agents (MTAs), such as sorafenib [5, 6], lenvatinib [7], regorafenib [8], ramucirumab [9], and cabozantinib [10]. These MTAs were demonstrated to have survival benefit noninferior to sorafenib or over placebo. On the other hand, immune checkpoint inhibitors (ICIs) are a promising therapy for unresectable HCC and have been shown to possess durable antitumor activity [11-14]. Antibodies against the programmed cell death 1 (PD-1) have shown prolonged antitumor responses in patients with advanced HCC and Child-Pugh class A liver function [11, 12]. The Food and Drug Administration approved the anti-PD-1 antibodies such as nivolumab [11] and pembrolizumab [12] as second-line therapies for patients with HCC whose disease progressed on prior sorafenib treatment. However, phase 3 clinical trials failed to show survival benefits of antiPD-1 antibodies for unresectable HCC in both first- and second-line settings $[15,16]$. These results revealed that approximately $30-36 \%$ of the patients with unresectable HCC were refractory to ICI monotherapy and had progressive disease $(\mathrm{PD})[15,16]$, which suggested the presence of potential refractory mechanisms against these agents.

Although all the mechanisms underlying the resistance to ICIs in patients with HCC remain unclear, activating mutations in the $\mathrm{Wnt} / \beta$-catenin pathway could induce the primary resistance $[17,18]$. It has also been reported that HCC with activating mutations in the $\mathrm{Wnt} / \beta$-catenin signaling block immune cell recruitment and result in poor outcomes following ICI treatment, sug- gesting a role of the $\mathrm{Wnt} / \beta$-catenin pathway activation in the establishment of an "immune cold" phenotype in HCC [17-19]. Harding et al. [20] have reported that activating mutations of the $\mathrm{Wnt} / \beta$-catenin pathway was associated with shorter progression-free survival (PFS) and no tumor response (progressive disease $100 \%$ ) in patients with HCC who were treated with ICIs. We have also found that combination score involving the status of $\mathrm{Wnt} / \beta$-catenin activation, degree of tumor infiltrating lymphocyte, and expression of programmed death-ligand 1 (PD-L1) well-stratify the PFS on anti-PD-1 monotherapy [21]. However, to assess the $\mathrm{Wnt} / \beta$-catenin pathway activation using proteomic and genomic analyses, HCC tissues are required for the analyses of gene expression and alterations of catenin beta-1 (CTNNB1), adenomatous polyposis coli, and AXIN1 and its downstream targets, such as glutamine synthetase. In order to avoid the invasive procedures involved in tissue sampling, an alternative noninvasive measure that reflects the activation of the canonical Wnt $/ \beta$-catenin pathway is required, which may predict tumor response or resistance to ICI treatment.

Gadolinium-ethoxybenzyl-diethylenetriamine (GdEOB-DTPA)-enhanced magnetic resonance imaging (MRI) is a gold-standard imaging modality with a high accuracy for diagnosing HCCs [22, 23]. Gd-EOB-DTPA is the liver-specific contrast agent for MRI uptake by hepatocytes through the organic anion transporting polypeptide 1B3 (OATP1B3) [24-27]. OATP1B3 was reported to be one of the downstream targets of the Wnt/ $\beta$ catenin signaling pathway [28]. The expression of OATP1B3 was demonstrated to be strongly associated with the activation of the $\mathrm{Wnt} / \beta$-catenin signaling pathway in HCC cases $[28,29]$. On the other hand, the immunohistochemical expression of OATP1B3 showed to be strongly correlated with iso-high intensity in hepatobiliary phase images of Gd-EOB-DTPA-enhanced MRI [27, 28]. Ueno et al. [28] reported that the activation of the $\mathrm{Wnt} / \beta$-catenin signaling pathway induced OATP1B3 messenger ribonucleic acid (mRNA) expression in HCC cells and also showed a strong association between OAT$\mathrm{P} 1 \mathrm{~B} 3$ expression and $\mathrm{Wnt} / \beta$-catenin signaling in human HCC tissues. Collectively, the activation status of the $\mathrm{Wnt} / \beta$-catenin signaling leads to the iso-high intensity of hepatobiliary phase images of Gd-EOB-DTPA-enhanced MRI, which should be an ideal predictive imaging marker for the poor response to ICIs [30].

To date, there are no reports on noninvasive biomarkers for ICIs in HCC, and there is an urgent need to carefully select patients with HCC who can benefit from ICIs. 
Fig. 1. Flow diagram of the objective patients with unresectableHCC. Of the unresectable HCC patients who received ICI therapy at the Kindai University Hospital from January 2015 to March 2020, published 18 patients met the inclusion criteria were selected. HCC, hepatocellular carcinoma; ICIs, immune checkpoint inhibitors; PD-1, programmed cell death 1; PDL1, programmed death ligand-1; CTLA-4, cytotoxic T-lymphocyte antigen 4; GdEOB-DTPA, gadolinium-ethoxybenzyldiethylenetriamine; MRI, magnetic resonance imaging.

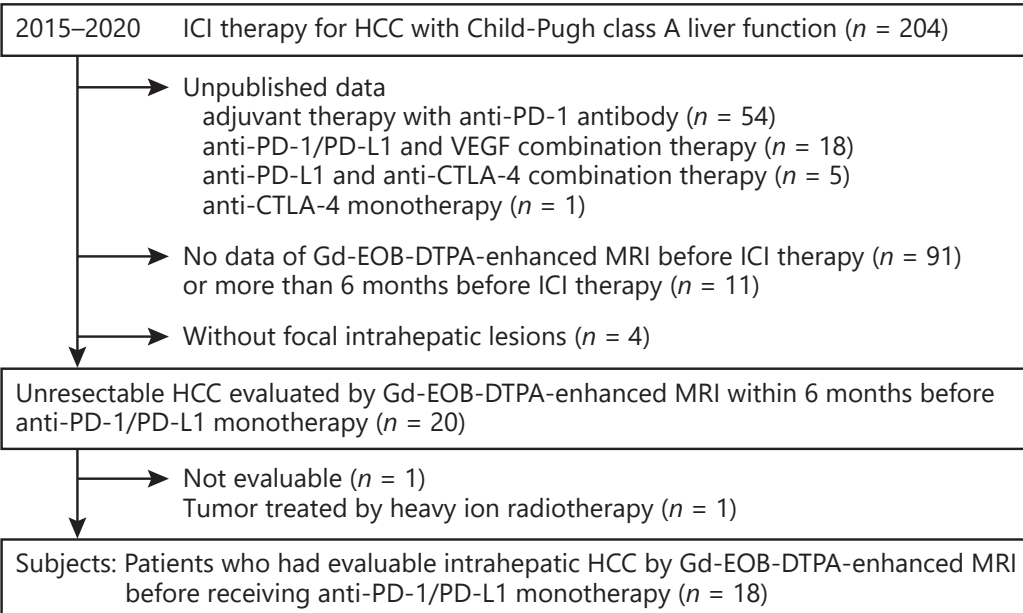

Unresectable HCC evaluated by Gd-EOB-DTPA-enhanced MRI within 6 months before anti-PD-1/PD-L1 monotherapy $(n=20)$

$\longrightarrow$ Not evaluable $(n=1)$

Tumor treated by heavy ion radiotherapy $(n=1)$

Subjects: Patients who had evaluable intrahepatic HCC by Gd-EOB-DTPA-enhanced MRI before receiving anti-PD-1/PD-L1 monotherapy $(n=18)$

In this study, we addressed this important issue and attempted to evaluate the response to anti-PD-1/PD-L1 monotherapy by the analysis of the intensity of HCC nodules on hepatobiliary phase images of Gd-EOB-DTPAenhanced MRI.

\section{Materials and Methods}

\section{Patients}

This single-institution retrospective cohort study enrolled 204 patients with unresectable HCC and Child-Pugh class A liver function who received ICIs from January 1, 2015 to March 31, 2020 at the Kindai University Hospital. The diagnosis of HCC was based on histological and/or radiological findings according to the guidelines proposed by the American Association for the Study of Liver Diseases [31].

The inclusion criteria were as follows: patients with performance status defined by the Eastern Cooperative Oncology Group [32] of 0 or 1, patients with intermediate to advanced stages of HCC treated with anti-PD-1/PD-L1 monotherapy, patients with evaluable viable intrahepatic lesions on Gd-EOB-DTPA-enhanced MRI examined within 6 months before the start of the PD-1/PDL1 antibody treatment, those with Child-Pugh class A liver function, and an expected survival time longer than 3 months. The exclusion criteria were as follows: patients with the high risk for esophageal and gastric variceal rupture, patients who refused to participate in this clinical research. The flow diagram for the selection of patients is shown in Figure 1. Eighteen patients with HCC met the criteria and were included in the analysis.

Dose reductions or interruptions were considered if the patients presented severe adverse events (AEs) defined in the Common Terminology Criteria for Adverse Events (CTCAE) v4 (grade 23) $[33,34]$. The Response Evaluation Criteria in Solid Tumors version 1.1 (RECIST v1.1) [35] was applied to assess the antitumor response to the target lesion. The tumor response was evaluated by
2 independent imaging experts specialized in HCC. This study was approved by the Institutional Review Board and Medical Ethics Committee of the Kindai University Hospital (IRB approval R02102), and written informed consent was obtained from all patients.

\section{MRI Protocol}

Gd-EOB-DTPA-enhanced MRI was performed using a superconducting magnet system of a 3T scanner (Intera Acheiva 3T; Philips Healthcare, Best, The Netherlands) or 1.5T scanner (1.5T Signa HDxt; GE Healthcare, Milwaukee, WI, USA) with an 8-channel body phased-array coil. Dynamic images using fat-suppressed T1-weighted gradient echo images were obtained before (pre-contrast), $14-30 \mathrm{~s}$ (arterial phase), $70 \mathrm{~s}$, and $3 \mathrm{~min}$ after intravenous administration of $0.025 \mathrm{mmol}$ per $\mathrm{kg}$ of body weight of gadoxetic acid (Primovist; Bayer Schering Pharma, Osaka, Japan) at a rate of $2.0 \mathrm{~mL} / \mathrm{s}$, followed by $20 \mathrm{~mL}$ of a saline flush. To capture the appropriate timing for arterial phase, a fluoroscopic bolus tracking method was used. In this method, the contrast agent was detected in the proximal abdominal aorta by visual inspection, and automatic detection was performed by placing the region of interest in the abdominal aorta (at the level of the subdiaphragm). The patient was instructed to hold his or her breath 5-10 s after the contrast agent was first detected, after which the arterial phase sequence was acquired. Hepatobiliary phase images were obtained 20 min after the injection of gadoxetic acid. MRI methods are summarized in online suppl. Table 1; for all online suppl. material, see www. karger.com/doi/10.1159/000518048. Two image sets with precontrast and hepatobiliary phase images were the focus of evaluation in this study.

\section{Assessment of Tumor Response to ICIs}

Among 20 patients who underwent treatment using anti-PD-1/ PD-L1 monotherapies, 18 were considered eligible for this study (shown in Fig. 1). The primary endpoint was PFS and the secondary endpoints were overall survival (OS), objective response rate (ORR), time to nodular progression (TTnP, $\geq 20 \%$ increase as compared with baseline) and AEs. 
Laboratory tests were performed every 2-4 weeks, and dynamic computed tomography or Gd-EOB-DTPA-enhanced MRI was performed every 4-8 weeks. In case of treatment discontinuation owing to the occurrence of AEs, laboratory tests and diagnostic imaging were repeated every 4-8 weeks until disease progression was observed. The disease control rate (DCR) and ORR were determined based on the best response observed during the treatment and observation periods with anti-PD-1/PD-L1 antibody. For safety assessment, AEs were assessed according to the CTCAE v4 [36]. Among these, 86 hypervascular intrahepatic HCC nodules were depicted using Gd-EOB-DTPA-enhanced MRI by 1 researcher (T.A. with 10 years of experience in hepatology). These nodules were evaluated by 2 independent researchers (K.U. with 25 years of experience in hepatology and M.T. with 25 years of experience in abdominal radiology, especially in liver imaging). Therefore, previously treated nodules, such as transcatheter arterial chemo-embolization and heavy particle beam, were excluded, and the remaining 68 nodules were eligible for analysis. Regions of interest (ROIs) were determined to measure the signal intensities (SI) of the intrahepatic HCC nodules and background liver. Measurements of SI of the intrahepatic HCC nodules were performed at the axial level where the nodule was largest and by placing a round ROI most closely approximating the entire nodule. Additionally, 3 ROIs on areas of background liver parenchyma were placed free of focal hepatic lesions, major branches of the portal or hepatic vein, or imaging artifacts, making sure to place the ROIs at the same positions on the liver parenchyma for each patient. The mean of the 3 ROI measurements was recorded as the SI of the parenchyma in each phase. ROIs of nodules at least 40 (average 150, range $40-1,300) \mathrm{mm}^{2}$ were positioned. The relative enhancement of nodules compared with that of the liver parenchyma was calculated as follows by 2 independent researchers (M.T. and K.S. with 19 years of experience in abdominal radiology, especially in liver imaging), and the average of the 2 values was used for this study. [28, 37]: the relative intensity ratio $($ RIR $)=$ SInod/SIpar, where SInod is the SI from the nodule and SIpar is that from the liver parenchyma; the relative enhancement ratio (RER) was calculated as RIRHBP/RIRpre, where RIRHBP is RIR in the hepatobiliary phase images and RIRpre is the RIR before the contrast enhancement. Nodules with the activation of the $\mathrm{Wnt} / \beta$-catenin signaling pathway reportedly showed an increase in RER; the optimum RER cutoff point for the determination of $\mathrm{Wnt} / \beta$-catenin activation was set as 0.90 according to a previous report by Ueno et al. [28]. HCC nodules with higher enhancement on hepatobiliary phase were defined as an RER $\geq 0.90$ and hypointense HCC nodules as an RER $<0.90$. With this cutoff point, the sensitivity, specificity, and accuracy of prediction of Wnt/ bcatenin-activated HCC were $78.9 \%, 81.7 \%$, and $81.2 \%$, respectively [28].

We evaluated 68 intrahepatic HCC nodules; the Gd-EOB-DTPA-enhanced MRI or dynamic computed tomography examination immediately before ICI treatment was defined as the observation start time, and the maximum diameter of baseline target tumors was measured. The maximum tumor diameter was measured by RECIST v1.1 at each periodic imaging examination, and the nodular progression rate from the baseline was calculated. The time when the tumor progression rate was $20 \%$ or more was defined as nodular PD.

\section{Clinical and Laboratory Evaluation}

The following test values were collected from medical records within 2 weeks before the initiation of ICI treatments: weight, height, body mass index, platelet counts, prothrombin time-international normalized ratio, serum albumin, total bilirubin, aspartate aminotransferase, alanine aminotransferase, $\gamma$-glutamyltransferase, $\alpha$-fetoprotein (AFP), and des- $\gamma$-carboxy prothrombin (DCP). The fibrosis-4 (FIB-4) index was calculated as age (years) $\times$ aspartate aminotransferase $(\mathrm{IU} / \mathrm{L}) /\left(\right.$ platelet count $\left(10^{9} / \mathrm{L}\right) \times \sqrt{ }$ alanine aminotransferase [IU/L]) [38].

\section{Statistical Analyses}

Statistical analyses were performed using the IBM Statistical Package for the Social Sciences statistics version 22.0 (IBM, Armonk, NY, USA). A 2-sided $p$ value of $<0.05$ was considered to indicate statistically significant difference in all analyses. Continuous variables were expressed as median (range), as appropriate. The Kaplan-Meier method and Cox proportional hazards regression models were applied to compare patient survival. When evaluating each nodule, the proportion of nodular PD was compared by RER ratio, and the $\chi^{2}$ test or Fisher's exact test was performed. The TTnP was tested by the Kaplan-Meier curve (log-lank test).

\section{Results}

\section{Patient Characteristics}

Characteristics of patients at the start of anti-PD-1/ PD-L1 monotherapy are summarized in Table 1. The median age of patients was 69 (range, 22-79) years, and $72 \%(13 / 18)$ of the patients were males. In this cohort study, $38.9 \%$ (7/18) of the patients were negative for hepatitis B surface antigen (HBsAg) and hepatitis C antibody, and further, $38.9 \%$ (7/18) were positive for hepatitis $\mathrm{C}$ antibody and $22.2 \%(4 / 18)$ were positive for HBsAg. The median FIB-4 index was 4.01 (range, 0.78-18.02), with $50.0 \%$ (9/18) of the patients having a FIB-4 index $>4.00$. Five of 18 patients (27.8\%) were classified as Barcelona clinic liver cancer (BCLC) stage B and $72.2 \%$ $(13 / 18)$ of the patients were BCLC stage C. Vascular invasion and extrahepatic spread were observed in $16.7 \%$ $(3 / 18)$ and $55.6 \%(10 / 18)$ patients, respectively. The baseline median AFP level was 1,957.5 (range, 4.0$52,190) \mathrm{ng} / \mathrm{mL} ; 55.6 \%$ (10/18) of patients showed serum AFP level $\geq 400 \mathrm{ng} / \mathrm{mL}$. The median DCP level was 804 (range, 93-24,227) $\mathrm{mAU} / \mathrm{mL}$, with $27.8 \%(5 / 18)$ of the patients showing a serum DCP level $\geq 400 \mathrm{mAU} / \mathrm{mL}$. Of the 18 patients, 8 received systemic therapy such as sorafenib before ICI treatment.

Eight patients (44\%) showed at least 1 HCC nodule with higher enhancement on the hepatobiliary phase of Gd-EOB-DTPA-enhanced MRI, whereas 10 patients (56\%) did not have any higher enhancement HCC nodules on the hepatobiliary phase images. Comparisons of patient characteristics are shown in Table 1. Among pa- 
Table 1. Patient characteristics at baseline

\begin{tabular}{|c|c|c|c|c|}
\hline Patient characteristic & $\begin{array}{l}\text { All patients } \\
(N=18)\end{array}$ & $\begin{array}{l}\text { Patients with higher } \\
\text { enhancement HCC } \\
\text { nodules }(n=8)\end{array}$ & $\begin{array}{l}\text { Patients with } \\
\text { hypointense HCC } \\
\text { nodules }(n=10)\end{array}$ & $p$ value \\
\hline Gender, male, $n(\%)$ & $13(72)$ & $6(75)$ & $7(70)$ & NS \\
\hline $\mathrm{HCV} / \mathrm{HBV} / \mathrm{NBNC}$ & $7 / 4 / 7$ & $4 / 1 / 3$ & $3 / 3 / 4$ & NS \\
\hline ECOG PS, $0 / 1$ & $18 / 0$ & $8 / 0$ & $10 / 0$ & NS \\
\hline Tumor number, median & $5(1$, many $)$ & $7(2$, many $)$ & $3(1,6)$ & 0.077 \\
\hline BCLC stage, $0-\mathrm{A} / \mathrm{B} / \mathrm{C}$ & $0 / 5 / 13$ & $0 / 5 / 3$ & $0 / 0 / 10$ & 0.009 \\
\hline Vascular invasion, $n(\%)$ & $3(17)$ & $1(13)$ & $2(20)$ & NS \\
\hline Extrahepatic spread, $n(\%)$ & $10(56)$ & $2(25)$ & $8(80)$ & 0.077 \\
\hline Child-Pugh score, $5 \mathrm{~A} / 6 \mathrm{~A}$ & $10 / 8$ & $4 / 4$ & $6 / 4$ & NS \\
\hline ALBI score, median & $-2.55(-3.24,-1.63)$ & $-2.35(-3.23,-1.62)$ & $-2.76(-3.18,-1.87)$ & NS \\
\hline Baseline DCP, median, mAU/mL & $804(93,24,227)$ & $572(93,18,340)$ & $869(144,24,227)$ & NS \\
\hline
\end{tabular}

Values were expressed as median (range). HCV, hepatitis C virus; HBV, hepatitis B virus; NBNC, HBV and HCV negative; BCLC, Barcelona clinic liver cancer; ALBI grade, albumin-bilirubin grade; FIB-4 index, fibrosis-4 index; AFP, $\alpha$-fetoprotein; DCP, Des- $\gamma$ carboxy prothrombin; HCC, hepatocellular carcinoma.

tients with at least 1 higher enhancement HCC nodule, $13 \%(1 / 8)$ had vascular invasion, and 25\% (2/8) had extrahepatic spread; BCLC stage C was $38 \%$ (3/8), which was significantly lower than those without higher enhancement HCC nodules, where all patients were classified as BCLC stage C ( $p=0.009)$.

\section{Administration of PD-1/PD-L1 Monotherapy}

At the data cutoff date (June 30,2020), the median duration of follow-up was 26.3 (95\% confidence interval [CI]: 13.8-38.8) months (Kaplan-Meier estimate). During the observation period, 12 patients $(66.7 \%)$ died because of HCC progression. Patients received immunotherapy either as a standard of care or in clinical trials. Of the 18 patients, 14 received anti-PD-1 monotherapy, and 4 patients received anti-PD-L1 monotherapy. The median treatment duration with anti-PD-1/PD-L1 antibody was 3.9 (range, 1.3-40.6) months. Treatment was discontinued in 17 patients due to disease progression $(n=14$, $77.8 \%)$, treatment-related AEs $(n=2,11.1 \%)$, or switching to transcatheter arterial chemoembolization $(n=1$, $5.6 \%)$. Only 1 (5.6\%) of the 18 patients with a partial response was still undergoing ICI treatment at the data cutoff.
ORR and PFS according to RER in the Hepatobiliary

Phase of Gd-EOB-DTPA-Enhanced MRI

Among the patients evaluable for tumor response, the ORR and DCR were $22.2 \%(4 / 18)$ and 55.6\% (10/18), respectively. The best responses evaluated were as follows: $0 \%(n=0)$ for complete response (CR), $22.2 \%(n=4)$ for partial response (PR), 33.3\% $(n=6)$ for stable disease, and $44.4 \%(n=8)$ for PD (online suppl. Table 2$)$. The median PFS from the start of anti-PD-1/PD-L1 antibody therapy in all patients was 4.23 (95\% CI: 0.42-8.05) months and the median OS was 26.3 (95\% CI: 12.8-38.8) months.

Among patients with higher enhancement HCC nodules on the hepatobiliary phase images, the ORR and DCR were $12.5 \%(1 / 8)$ and $37.5 \%(3 / 8)$, respectively. On the contrary, the ORR and DCR were $30.0 \%(3 / 10)$ and $70.0 \%(7 / 10)$, respectively among patients with hypointense HCC nodules (online suppl. Table 2). There was no statistically significant difference in ORR and DCR between both groups. The median PFS was 2.7 (95\% CI: 1.4-4.0) months in patients with higher enhancement HCC nodules in the hepatobiliary phase $(n=8)$ and 5.8 months (95\% CI: 0.0-18.9) in patients with hypointense HCC nodules $(n=10)(p=0.007$; shown in Fig. 2). A Cox regression analysis adjusted for age $\geq 65$ years, hepatitis virus infection, ALBI grade, serum AFP level $\geq 400 \mathrm{ng} /$ 


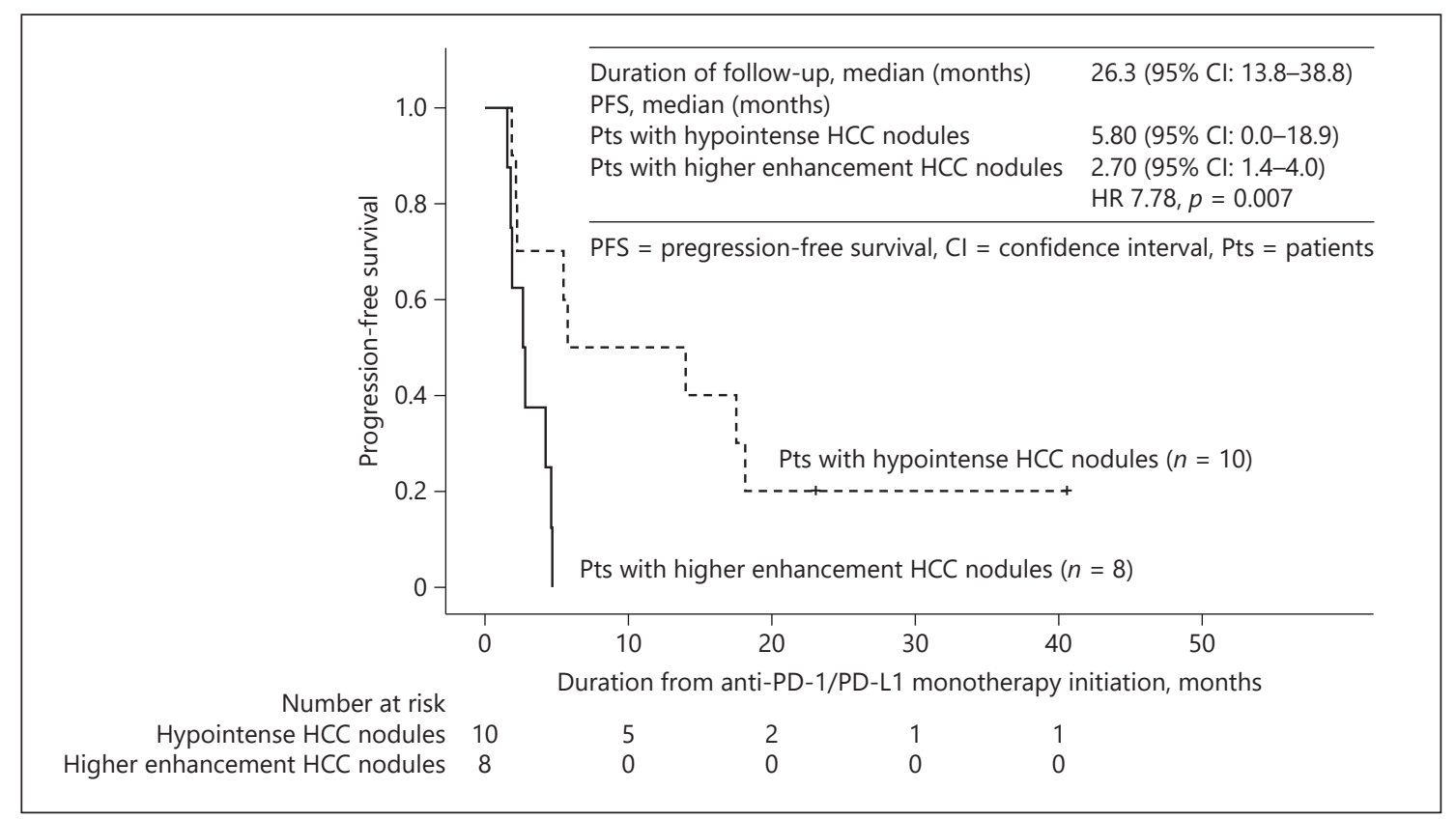

Fig. 2. Kaplan-Meier analysis of PFS. The median PFS was 2.70 months in the patients with higher enhancement HCC nodules on hepatobiliary phase, and 5.80 months in the patients without high- er enhancement HCC nodules $(p=0.007)$. PFS, progression-free survival; HCC, hepatocellular carcinoma.
Table 2. Results of Cox regression analysis (forced entry method)

\begin{tabular}{llll}
\hline Category & Hazard ratio & $95 \%$ CI & $p$ value \\
\hline HCV infection & 1.85 & $0.30-11.4$ & 0.509 \\
HBV infection & 2.96 & $0.34-26.1$ & 0.329 \\
Age $\geq 65$ years & 1.30 & $0.31-5.47$ & 0.723 \\
ALBI grade & 2.61 & $0.51-13.4$ & 0.250 \\
AFP $\geq 400$ & 1.00 & $0.20-5.04$ & 0.997 \\
DCP $\geq 800$ & 1.38 & $0.15-12.9$ & 0.779 \\
RER $\geq 0.90$ & 7.78 & $1.59-38.1$ & 0.011 \\
\hline
\end{tabular}

Of the 18 cases, 4 were censored and 14 had progressive disease. True clinical outcome (dependent variables) ware PFS or time to progression (TTP) and cox regression analysis was performed with $\mathrm{HCV}, \mathrm{HBV}$, age $\geq 65$, ALBI grade, AFP $\geq 400, \mathrm{DCP} \geq 800$, and RER $\geq 0.90$ as independent variables. $\mathrm{HCV}$, hepatitis $\mathrm{C}$ virus; $\mathrm{HBV}$, hepatitis B virus; AFP, a-fetoprotein; ALBI, albumin bilirubin; DCP, des- $\gamma$-carboxy prothrombin; RER, relative enhancement ratio; CI, confidence interval; PFS, progression-free survival.

$\mathrm{mL}$, and DCP level $\geq 800 \mathrm{mAU} / \mathrm{mL}$ showed that the hazard ratio was 7.78 (95\% CI: $1.59-38.1, p=0.011)$ in patients having higher enhancement HCC nodules defined by RER $\geq 0.90$ (Table 2). The median OS from the start of anti-PD-1/PD-L1 checkpoint therapy was 29.0 (95\% CI:
12.2-45.8) months in the patients with at least 1 higher enhancement HCC nodules, and 24.5 (95\% CI: 13.135.9) months in the patients with hypointense HCC nodules $(p=0.523$; online suppl. Fig. 1$)$.

\section{Time to Nodular Progression and PD Rate in Each Nodule}

A total of 68 hypervascular HCC nodules in 18 patients without a past treatment history were retrospectively examined for antitumor response against corresponding nodules. The characteristics of these nodules are shown in Table 3 and Figure 3. HCC nodules with higher enhancement on the hepatobiliary phase images were observed in 23 of 68 nodules, and hypointense HCC nodules were observed in 45 of 68 nodules. We used the average RER values measured by 2 independent radiologists. By using the average RER of 2 readers, most of the discordances between visual assessment and RER on nodules were finally fixed, but discrepancy was still observed in a few nodules with heterogeneous areas and fat deposits. The median tumor size was 1.5 (range, $0.8-4.0$ ) $\mathrm{cm}$ for higher enhancement HCC nodules and 1.7 (range, 0.810.4) $\mathrm{cm}$ for hypointense HCC nodules. We calculated the contrast-to-noise ratio for the hepatobiliary phase and enhancement ratio, which were used to evaluate the 

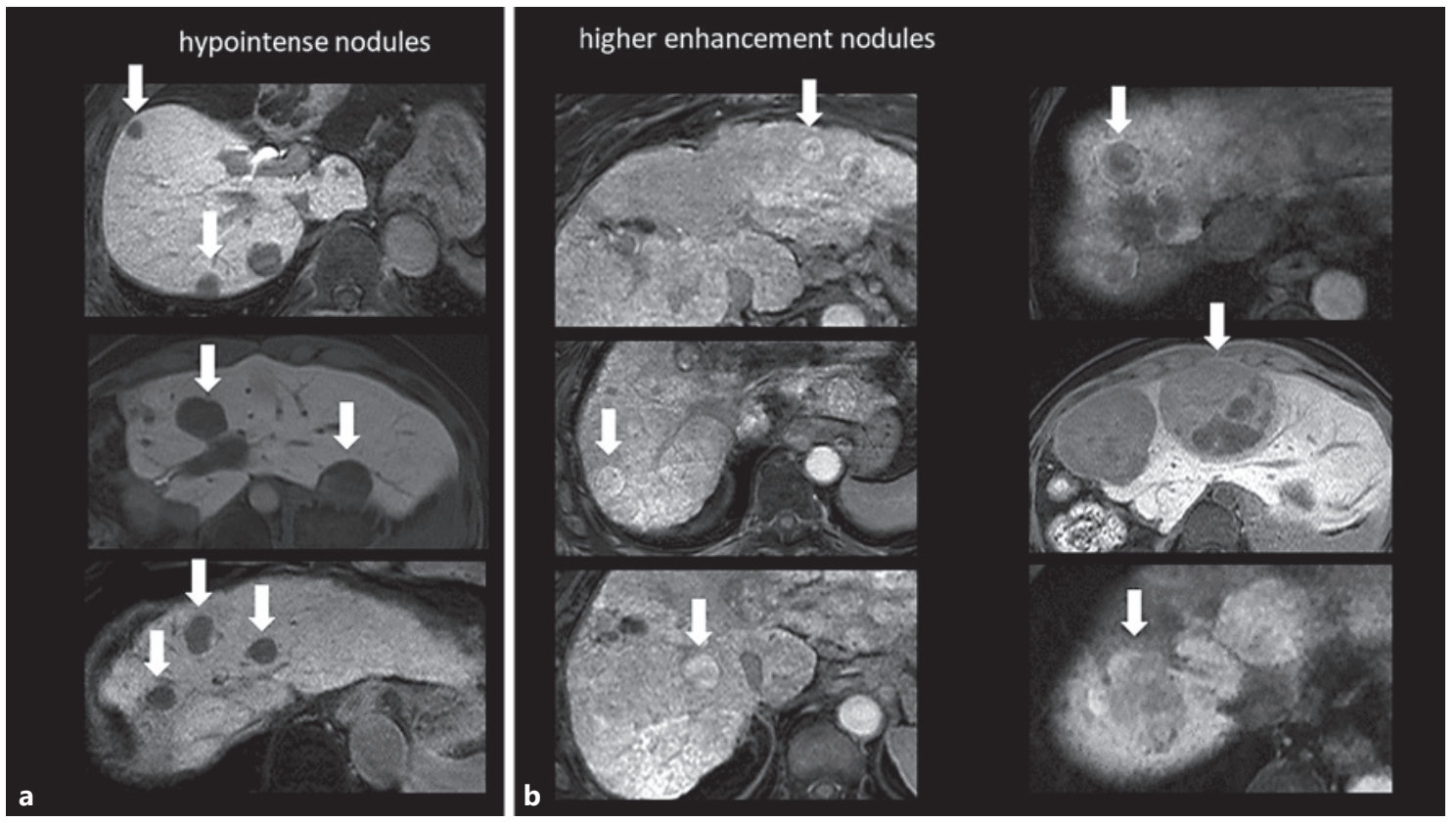

Fig. 3. Classification of tumor image based on RER and visual signal intensity. Typical nodular images on hepatobiliary phase of Gd-EOB-DTPA-enhanced MRI were presented. We defined HCC nodules with RER $<0.9$ as hypointense nodules, HCC nodules with RER $>0.9$ as higher enhancement nodules. a Typical hypointense HCC nodules with RER <0.9: this enhancement pattern was seen in a typical HCC, clearly lower signal intensity than the background liver on hepatobiliary phase. b Typical higher enhancement HCC nodules with RER >0.9: this enhancement pattern was seen in a green hepatoma, clearly higher signal intensity than the

background liver on hepatobiliary phase of Gd-EOB-DTPA-enhanced MRI. Some of them, heterogeneous intensity HCC nodules have been including, mixed regions of slightly higher signal intensity on the hepatobiliary phase. It is difficult to measure RER in this type of tumors. In this study, RER was measured by setting ROIs over the entire tumor. Gd-EOB-DTPA, gadolinium-ethoxybenzyl-diethylenetriamine; MRI, magnetic resonance imaging; HCC, hepatocellular carcinoma; RER, relative enhancement ratio; ROI, region of interest.

Table 3. Clinical characteristics of intrahepatic HCC nodules

\begin{tabular}{lccc}
\hline Feature & $\begin{array}{l}\text { Higher enhancement } \\
\text { HCC nodules }(n=23)\end{array}$ & $\begin{array}{l}\text { Hypointense } \\
\text { HCC nodules }(n=45)\end{array}$ & $p$ value \\
\hline Tumor size, median, cm & $1.5(0.8,4.0)$ & $1.7(0.8,10.4)$ & NS \\
RER, ${ }^{*}$ median & $1.04(0.92,6.32)$ & $0.72(0.17,0.89)$ & $<0.001$ \\
CNR on HBP, ${ }^{* *}$ median & $3.6(-4.8,14.4)$ & $11.9(4.0,42.4)$ & 0.031 \\
Enhancement ratio, ${ }^{* *}$ median & $0.16(-0.62,2.99)$ & $0.012(-0.51,1.70)$ & 0.039 \\
ADC, median & $1.16(0.61,1.72)$ & $1.14(0.61,2.06)$ & NS \\
\hline
\end{tabular}

RER was measured by 2 independent radiologists and the average of the 2 values was used for the study. HCC nodules with higher enhancement on hepatobiliary phase were defined as those showing RER $\geq 0.90$. We express the inspection value in median (range). RER, relative enhancement ratio; RIR, relative intensity ratio; CNR on HBP, contrast-to-noise ratio on hepatobiliary-phase; ADC, apparent diffusion coefficient; HCC, hepatocellular carcinoma; SD, standard deviation; SI, signal intensity; SD background, standard deviation of the background noise; SInod, nodule SI; SIpar, liver parenchyma SI. * RER: RIR(HBP)/RIR(pre), where RIR(HBP) is RIR in the hepatobiliary-phase images and RIR(pre) is the precontrast RIR. RIR = SInod/SIpar. ${ }^{* *}$ CNR on HBP: CNR = (SInod - SIpar)/SD background .*** Enhancement ratio: the enhancement ratio during the hepatobiliary phase was calculated as (SIpost - SIpre)/SIpre. 


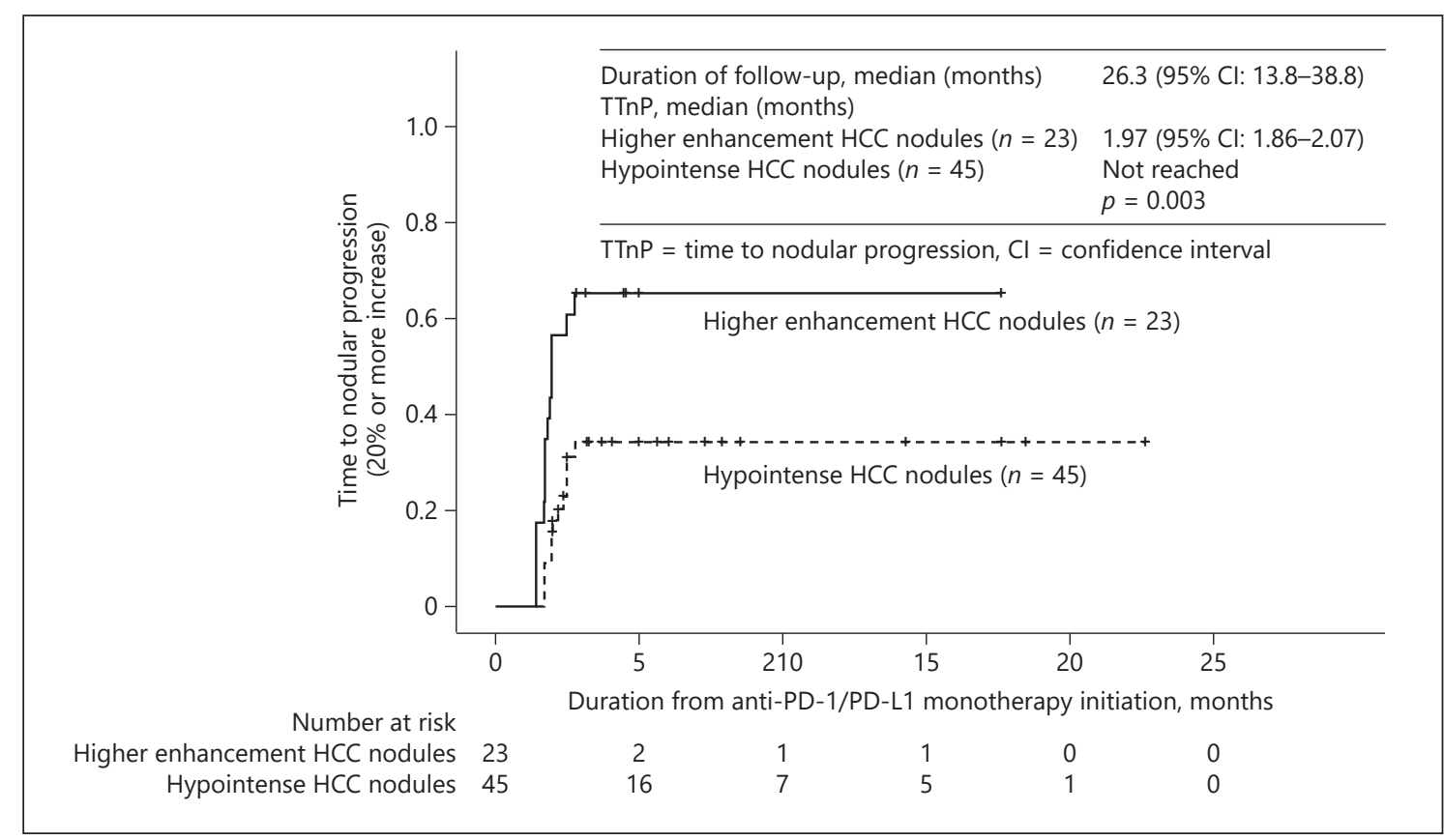

Fig. 4. Kaplan-Meier analysis of time to nodular progression. The time to nodular progression of each nodule above the specified threshold (20\% for RECIST v1.1) was 1.97 (95\% CI: 1.86-2.07) months in HCC nodules with higher enhancement. It was not reached in hypointense HCC nodules ( $p=0.003)$. RECIST v1.1, Response Evaluation Criteria in Solid Tumors version 1.1; HCC, hepatocellular carcinoma. signal value, instead of RER, and we observed a good correlation between these values and RER.

The waterfall plots for each higher enhancement nodule and hypointense nodule classified by RER were shown in online suppl. Figure 2. The proportion of nodular PD, which defined as a tumor size increase above the specified threshold (20\% per RECIST v1.1), was $43.5 \%(10 / 23)$ on higher enhancement HCC nodules and 33.3\% (15/45) on hypointense HCC nodules. The time to progression of each nodule above the specified threshold (20\% per RECIST v1.1), TTnP, was 1.97 (95\% CI: 1.86-2.07) months in HCC nodules with higher enhancement on the hepatobiliary phase and not reached in hypointense HCC nodules ( $p=0.003$; shown in Fig. 4). Eight of the 68 nodules are histologically evaluated with tumor biopsy just before the anti-PD-1/PD-L1 treatment. The nuclear staining of $\beta$-catenin was detected in 3 of 8 nodules. Two of 3 nodules showing $\beta$-catenin staining fell into PD. We present typical examples of nodules with increased tumor diameter for RER $>0.90$ and nodules with decreased tumor diameter for RER $<0.90$ (Fig. 5a, b). We also experienced a unique case that carry different RER value nodules, and each of these nodules responded differently for anti-PD-1/PD-L1 monotherapy (Fig. 6).

\section{Safety}

Observed AEs of any grade are described in Table 4. AEs were observed in 15 patients (83.3\%), with grade 3 or 4 AEs occurring in $6(33.3 \%)$ patients. The most common AE was pruritus $(n=8,44.4 \%)$, which is considered a grade 2 or low-grade AEs. Liver dysfunction was the second most common $\mathrm{AE}(n=5,27.8 \%)$, and $1(5.6 \%)$ of them had grade 4 liver injury and required administration of systemic corticosteroids. Upper gastrointestinal bleeding from esophageal varices occurred in $1(5.6 \%)$, nasal bleeding occurred in 1 (5.6\%), upper airway bleeding occurred in 1 (5.6\%), bacteremia occurred in 2 (11.1\%), and fulminant type 1 diabetes, adrenal insufficiency, and hypothyroidism occurred in 1 patient (5.6\%). The reasons for treatment discontinuation were gastrointestinal bleeding $(n=1,5.6 \%)$ and severe liver injury $(n=$ $1,5.6 \%)$.

\section{Discussion}

ICIs have been approved for use in different types of malignancies, including melanoma, lung cancer, colorectal cancer, and HCC [39-42]. Monotherapy with ICIs, 

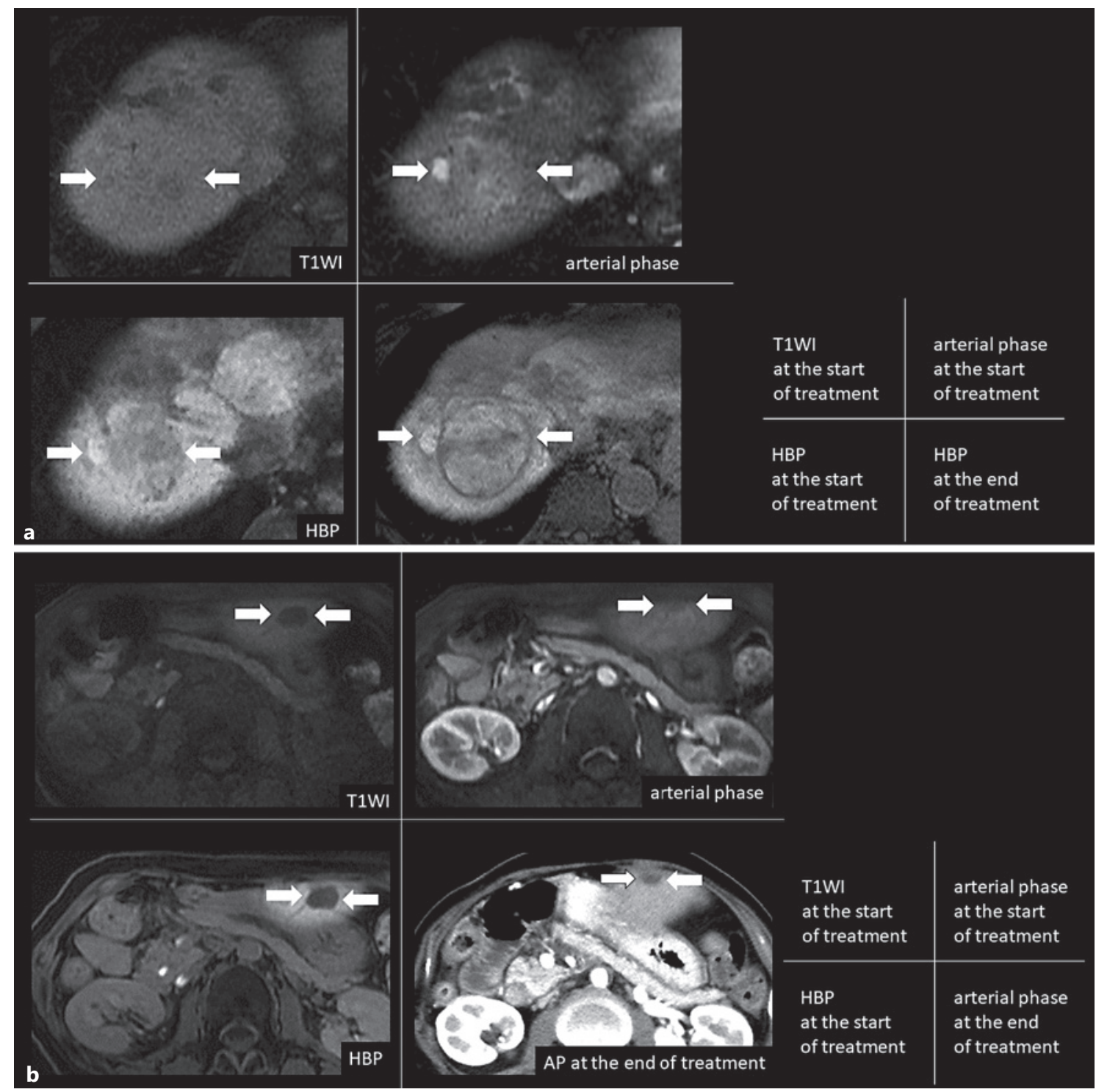

Fig. 5. a Typical treatment outcome in higher enhancement HCC nodule with tumor growth 60s male with Child-Pugh score 5, HCV positive (post-SVR) with alcohol intake. The average RER of the 2 readers was 1.044 in the large intrahepatic nodule of segment 8. After discontinuing sorafenib as the 1st line, anti-PD-L1 monotherapy was started as the 2 nd line therapy, and the nodule of segment 8 increased from $40 \mathrm{~mm}$ to $58 \mathrm{~mm}$ in 1.57 months, resulting in PD. b A typical treatment outcome in hypointense HCC nodule with tumor reduction 50s female with Child-Pugh score 5, HCV and HBV negative patient. The average RER of the 2 readers was

specifically those targeting PD-1 or PD-L1, has revolutionized the treatment of unresectable HCC $[11,12]$ because approximately $15-18 \%$ of patients achieve a partial or complete response, many of which show durable responses $[15,16]$.

EOB-MRI as a Noninvasive Responsive Marker of Anti-PD-1/PD-L1 Therapy
0.703 in the small intrahepatic nodule of segment 2. After discontinuing sorafenib as the 1 st line, anti-PD-1 monotherapy was started as the 2 nd line therapy, and the nodule of segment 2 decreased from $14 \mathrm{~mm}$ to $5 \mathrm{~mm}$ in 4 months, resulting in PR. RER, relative enhancement ratio; $\mathrm{HCV}$, hepatitis $\mathrm{C}$ virus; $\mathrm{HBV}$, hepatitis $\mathrm{B}$ virus; SVR, sustained virological response; RIR, the relative intensity ratio; PD-L1, programmed death-ligand 1; PD, progressive disease; $\mathrm{PR}$, partial response; HBP, hepatobiliary phase; T1WI, T1-weighted image; HCC, hepatocellular carcinoma; $\mathrm{PD}-1$, programmed cell death protein 1 .

Based on clinical trials using ICI monotherapies, a subset of HCC could respond to this type of agent, while others showed early PD. Considering the antitumor mechanism of anti-PD-1/PD-L1 antibody, it is reasonable to speculate that the efficacy of ICIs would be affected by the 

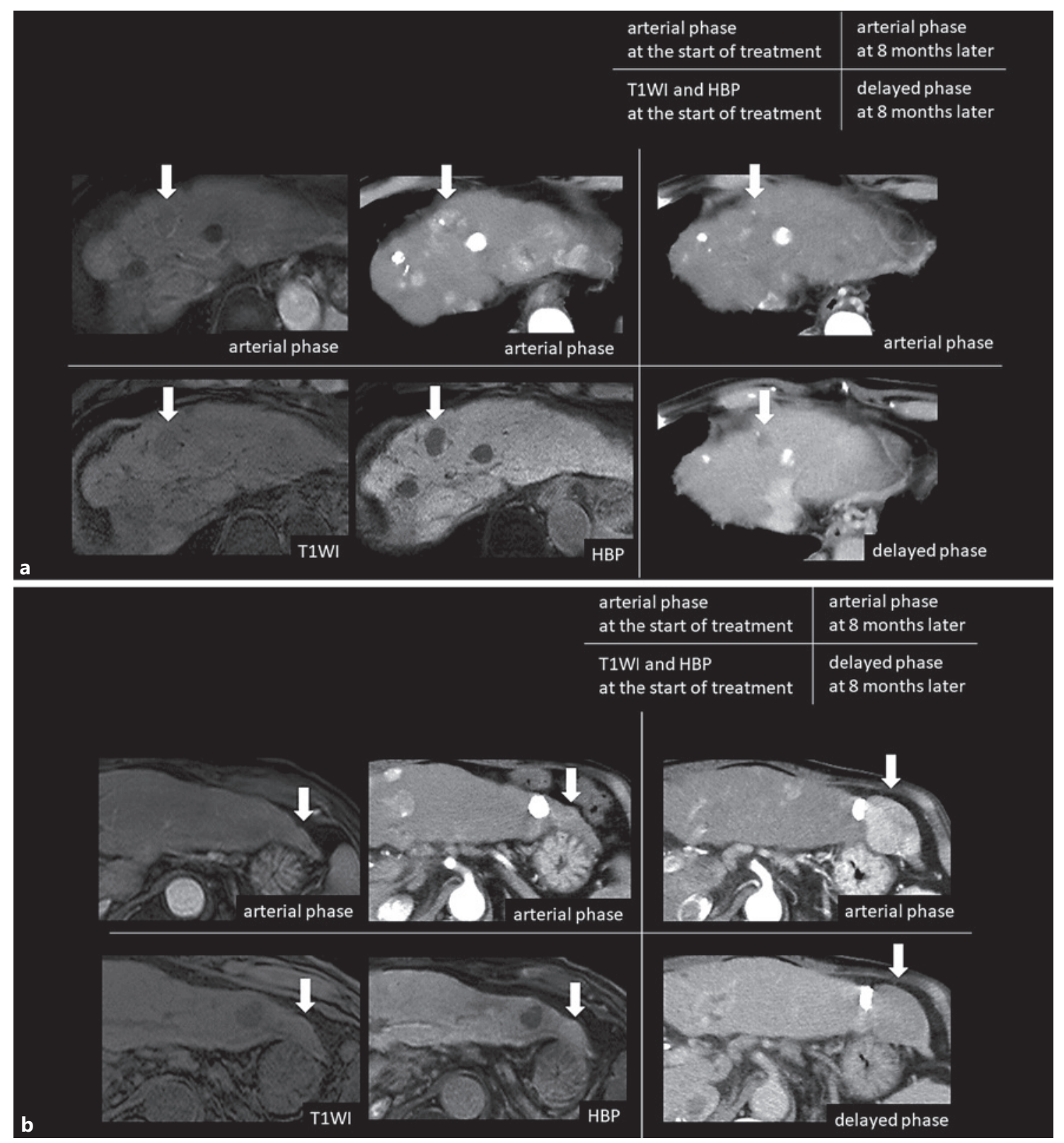

Fig. 6. Different response to anti-PD-1/PD-L1 monotherapy in the same case. We experienced different responses to anti-PD-1/PDL1 monotherapy when the same case had different RER nodules. 70 s male with Child-Pugh score $5, \mathrm{HCV}$ positive (post-SVR) patient. After discontinuing sorafenib and other therapies as the 1st to 3 rd line, anti-PD-1 monotherapy was started as the 4 th line therapy. SNR of the background liver in T1WI was 35.9, and SNR of background liver in hepatobiliary phase was 69.6. The SNR change ratio was $93.9 \%$, which was sufficient to evaluate that the liver parenchyma was well contrasted. a The average RER of the 2 readers was 0.726 , then the nodule of segment 4 decreased from $35 \mathrm{~mm}$ to $5 \mathrm{~mm}$ in 15 months, resulting in PR. b The average RER of the 2 readers was 1.037, then the nodule of segment 2 increased from 10 $\mathrm{mm}$ to $48 \mathrm{~mm}$ in 15 months, resulting in PD. RER, relative enhancement ratio; $\mathrm{HCV}$, hepatitis $\mathrm{C}$ virus; $\mathrm{SVR}$, sustained virological response; $\mathrm{RIR}$, the relative intensity ratio; $\mathrm{PD}-\mathrm{L} 1$, programmed death-ligand 1; PR, partial response; $\mathrm{PD}$, progressive disease; $\mathrm{HBP}$, hepatobiliary phase; T1WI, T1-weighted image; SNR, signal-tonoise ratio; $\mathrm{PD}-1$, programmed cell death 1 . 
Table 4. AEs of anti-PD-1/PD-L1 antibody therapy

\begin{tabular}{lll}
\hline \multirow{2}{*}{ AE } & \multicolumn{2}{l}{ All patients $(n=18), n(\%)$} \\
\cline { 2 - 3 } & any grade & grade 3/4 \\
\hline Pruritus & $8(44)$ & $0(0)$ \\
Proteinuria & $1(6)$ & $0(0)$ \\
Diarrhea & $1(6)$ & $0(0)$ \\
Decreased appetite & $0(0)$ & $0(0)$ \\
Fatigue & $0(0)$ & $0(0)$ \\
Liver injury or failure & $5(28)$ & $1(6)$ \\
Encephalopathy & $0(0)$ & $0(0)$ \\
Jaundice & $1(6)$ & $1(6)$ \\
Hypoalbuminemia & $0(0)$ & $0(0)$ \\
Ascites & $2(11)$ & $0(0)$ \\
Peripheral edema & $1(6)$ & $0(0)$ \\
Bleeding or hemorrhage & $3(17)$ & $1(6)$ \\
Hypothyroidism & $1(6)$ & $0(0)$ \\
Arthritis & $1(6)$ & $0(0)$ \\
Hyperglycemia & $1(6)$ & $1(6)$ \\
Adrenal insufficiency & $1(6)$ & $1(6)$ \\
Infection & $2(11)$ & $2(11)$ \\
\hline
\end{tabular}

PD-1, programmed cell death protein 1; PD-L1, programmed death-ligand 1 ; AE, adverse event.

tumor immune microenvironment and gene mutation status, such as $\mathrm{Wnt} / \beta$-catenin mutation. There are several factors associated with the primary resistance to $\mathrm{PD}-1$ and PD-L1 antibody, such as the loss of PD-1/PD-L1 expression by tumor cells or antigen-presenting cells $[43,44]$ and tumor immunogenicity that is mainly attributed to the tumor mutational burden, where microsatellite instability is one of the surrogate markers for predicting the efficiency of ICIs. It has been reported that approximately $11-37 \%$ of HCC have Wnt $/ \beta$-catenin mutation [45]. Activation of the canonical Wnt $/ \beta$-catenin signaling pathway in HCC decreases in chemokine (c-c motif) ligand 5 (CCL5) production, so the infiltration of CD8+ T cells into the tumor tissues by reducing the recruitment of CD103+ dendritic cells $[46,47]$, leading to the establishment of an "immune cold tumor" with the absence of antitumor cytotoxic $\mathrm{T}$ cell, leading to primary resistance to ICIs as one of the characteristics $[18,20,47]$. Other mechanisms of acquired resistance to $\mathrm{PD}-1$ and $\mathrm{PD}-\mathrm{L} 1$ antibodies include mutations in $\beta-2$ microglobulin that impair antigen presentation [48-50] and alterations in the Janus kinases, signal transducers, and activators of the transcription pathway that affect interferon- $\gamma$ signaling [51].

Kan et al. [52] described the complete genome sequencing of $88 \mathrm{HCC}$, and they found the Wnt/ $\beta$-catenin gene $(15.9 \%)$ and TP53 (35.2\%) to be the most commonly mutated genes, which were also considered as the 2 main oncogenic drivers for hepatocarcinogenesis. Then, it has been reported that TP53 was rarely positive in the $\mathrm{Wnt} / \beta$-catenin signaling-related marker-positive HCC [53]. Harding et al. [20] have reported that the activating mutations of CTNNB1 or AXIN1 was associated with progressive disease in $100 \%$, and showed a short median PFS in patients with HCC treated with ICIs. Based on the hypothesis that the activation status of the $\mathrm{Wnt} / \beta$-catenin signaling results in the enhancement of the hepatobiliary phase of Gd-EOB-DTPA-enhanced MRI [27-30], this study was conducted to predict the antitumor effect of ICIs for patients with unresectable HCC using the signal intensity of intrahepatic HCC nodules on the hepatobiliary phase of Gd-EOB-DTPA-enhanced MRI.

The frequency of higher enhancement HCC is about $10 \%$, which is smaller than that of $\mathrm{Wnt} / \beta$-catenin mutation in HCC. One reason is that population in which the mutation was evaluated may be different. HCC with Wnt/ $\beta$-catenin mutation is originally with a good prognosis, and $\mathrm{Wnt} / \beta$-catenin mutation has been reported to be $30 \%$ in only resectable cases. Its frequency in advanced stage HCC has not been clarified. Another reason is that suppressors of Wnt receptor are affected by methylation and therefore do not always match the reported frequency of gene mutations. It is necessary to compare it with the tumor biopsy, but in this study the target biopsy has not been sufficiently done, therefor further studies are needed to clarity on this point in the future.

The median PFS was significantly shorter in patients with higher enhancement HCC nodules on the hepatobiliary phase images than in those with hypointense HCC nodules (2.7 vs. 5.8 months, $p=0.007$ ). Comparing tumor factors, BCLC stage $\mathrm{C}$ was significantly more frequent and baseline AFP level and DCP level also tended to be higher in patients without any higher enhancement HCC nodules than in those with higher enhancement HCC nodules which is consistent with the observation by Kitao et al. $[27,53]$. It has been reported that HCC with higher enhancement on hepatobiliary phase of Gd-EOB-DTPAenhanced MRI were related to the good prognosis because of hepatocyte nuclear factor 4 alpha (HNF4a), which may work as a tumor suppressor gene and inhibit the progression of HCC [54]. After the BCLC stage and tumor marker level were adjusted for the analyses, the difference in PFS might be more prominent between the 2 groups.

We also detect significant difference in the analyses of 68 HCC nodules classified based on RER: TTnP is sig- 
nificantly shorter in HCC nodules with higher enhancement on the hepatobiliary phase images of Gd-EOB-DTPA-enhanced MRI than that in hypointense HCC nodules (1.97 months vs. not reached, $p=0.003)$. These results strongly support the notion that $\mathrm{Wnt} / \beta$-catenin activating mutations can be visualized on hepatobiliary phase of Gd-EOB-DTPA-enhanced MRI and HCC nodules with higher enhancement on hepatobiliary phase of Gd-EOB-DTPA-enhanced MRI could be an imaging biomarker to predict poor response to anti-PD-1/PD-L1 monotherapy [30]. However, although we find the association between Gd-EOB-DTPA-uptake and disease control on anti-PD-1/PD-L1 monotherapy, some nodules show discrepancy between tumor response to ICI and intensity on hepatobiliary phase of Gd-EOB-DTPAenhanced MRI. It is reported that transcription factor $\mathrm{HNF} 4 a$, which plays a role in maintaining the function of mature hepatocyte, can also induce the expression of OATP1B3, and a decrease in HNF4a during the de-differentiation of HCC may lead to the downregulation of OATP1B3, regardless of mutational status of $\mathrm{Wnt} / \beta$ catenin pathway, which results in the loss of Gd-EOBDTPA uptake [54]. From this point of view, it is conceivable that some HCC nodules may show discordance between Gd-EOB-DTPA uptake and Wnt/ $\beta$-catenin status. Further analyses should be required to understand the molecular background of the HCCs showing discordance between Gd-EOB-DTPA uptake and tumor response to ICI.

On the other hand, ICI-refractory HCCs often carry $\mathrm{Wnt} / \beta$-catenin mutations; it has been reported that the $\mathrm{Wnt} / \beta$-catenin activating mutations are highly correlated with fibroblast growth factor receptor 4 (FGFR4) [55]. In addition, it was shown that ORR and PFS by lenvatinib treatment were extremely favorable in FGFR4 expressed HCCs [55]. In this context, lenvatinib, which has a strong FGFR4 inhibitory activity, is likely to be very effective as a post-anti-PD-1/PD-L1 therapy for ICI refractory patients with HCC [56].

In this study, there is no significant difference in median OS (online suppl. Fig. 1). It has been reported that HCC with higher enhancement is originally low-grade malignancy and has a good prognosis [29, 54]. In addition, even if the effect on anti-PD-1/PD-L1 monotherapy is poor, the prognosis is extended by the natural course along with effects of post-treatment. Then, it may be difficult to make a significant difference in the OS.

Despite the promising results on evaluating RER using Gd-EOB-DTPA-enhanced MRI for ICI treatment, our study has several limitations. First, this study was retro- spective in nature and had a small sample size with imbalanced tumor factors. Second, there was no pathological proof, such as immunostaining of glutamine synthetase and $\beta$-catenin, or sequencing analysis of CTNNB1, adenomatous polyposis coli, and AXIN1. However, considering the establishment of noninvasive image-based biomarkers to predict the response to anti-PD-1/PD-L1 antibody, the data presented here should be quite informative for the selection of patients who are expected to be nonresponders to anti-PD-1/PD-L1 antibody in real-world clinical practice.

\section{Conclusion}

For patients with unresectable HCC and Child-Pugh class A liver function, RERs on the hepatobiliary phase of Gd-EOB-DTPA-enhanced MRI are noninvasive useful predictors of poor response to anti-PD-1/PD-L1 monotherapy. Patients with higher enhancement intrahepatic HCC nodules, which may reflect the activation of the $\mathrm{Wnt} / \beta$-catenin signaling pathway, could be good predictors of poor response to ICI treatments. Future largescale prospective studies are warranted to confirm our novel observation described in this report.

\section{Acknowledgement}

We would like to thank Editage (www.editage.com) for English language editing.

\section{Statement of Ethics}

IRB approval R02-102 (approved in 2020). This study was approved by the Institutional Review Board and Medical Ethics Committee of the Kindai University Hospital (IRB approval R02-102), and the written informed consent was obtained from all the patients.

\section{Conflict of Interest Statement}

T.A.: no relevant conflicts of interest to disclose. N.N. is an Editorial Board member of LIVER CANCER. K.U. has received Honoraria from Bayer AG, Eisai Co. Ltd., Merck Sharp \& Dohme, Eli Lilly and Company, Chugai Pharmaceutical Co., Ltd., Takeda Pharmaceutical Co., Ltd., Pfizer Inc., Otsuka Pharmaceutical Co., Ltd., Sumitomo Dainippon Pharma Co., Ltd., Taiho Pharmaceutical. Co. Ltd., EA Pharma Co., Ltd., AbbVie GK, and Kowa Co., Ltd. He has received consulting or advisory fees from Eisai Co. Ltd., Eli Lilly and Company, Chugai Pharmaceutical Co., Ltd., Takeda Pharmaceutical Co., Ltd., and Pfizer Inc. M.M.: no relevant conflicts of interest to disclose. H.C.: no relevant conflicts of interest 
to disclose. M.T.: no relevant conflicts of interest to disclose. S.H.: no relevant conflicts of interest to disclose. H.I.: no relevant conflicts of interest to disclose. Y.M.: no relevant conflicts of interest to disclose. A.Y.: no relevant conflicts of interest to disclose. K.S.: no relevant conflicts of interest to disclose. M.T.: no relevant conflicts of interest to disclose. M.K. has received grants from Taiho Pharmaceuticals, Chugai Pharmaceuticals, Otsuka, Takeda, Sumitomo Dainippon-Sumitomo, Daiichi Sankyo, AbbVie, Astellas Pharma, and Bristol-Myers Squibb. He has also received grants and personal lecture fees from Merck Sharpe and Dohme (MSD), Eisai, and Bayer, and is an adviser for MSD, Eisai, Bayer, BristolMyers Squibb, Eli Lilly, Chugai, AstraZeneca, and ONO Pharmaceuticals. He is an editor-in-chief of LIVER CANCER.

\section{Funding Sources}

This work was supported in part by a Grant-in-Aid for Scientific Research from the Japan Society for the Promotion of Science (KAKENHI: 16K 382, N. Nishida, and 18K07922, M. Kudo) and a grant from Smoking Research Foundation (N. Nishida).

\section{Author Contributions}

Conceptualization: M.K. and K.U.; methodology: M.K.; software: T.A.; validation: T.A.; formal analysis: T.A.; investigation: T.A. and M.T.; data curation, A.Y., K.U., M.M., H.C., M.T., S.H., H.I., and Y.M.; writing - original draft preparation: T.A. and N.N.; writing - review and editing: N.N. and M.K.; visualization: K.S. and M.T.; supervision: M.K.; and project administration: N.N. and M.K.

\section{Data Availability Statement}

All data generated or analysed during this study are included in this article and its online suppl. files. Further inquiries can be directed to the corresponding author.

\section{References}

1 Altekruse SF, Henley SJ, Cucinelli JE, McGlynn KA. Changing hepatocellular carcinoma incidence and liver cancer mortality rates in the United States. Am J Gastroenterol. 2014 Apr;109(4):542-53.

2 Torre LA, Bray F, Siegel RL, Ferlay J, LortetTieulent J, Jemal A. Global cancer statistics, 2012. CA Cancer J Clin. 2015 Mar;65(2):87108.

3 European Association for the Study of the Liver. EASL clinical practice guidelines: management of hepatocellular carcinoma. J Hepatol. 2018 Jul;69(1):182-236.

4 Lin L, Yan L, Liu Y, Qu C, Ni J, Li H. The burden and trends of primary liver cancer caused by specific etiologies from 1990 to 2017 at the global, regional, national, age, and sex level results from the Global Burden of Disease Study 2017. Liver Cancer. 2020 Sep;9(5):563-82.

5 Llovet JM, Ricci S, Mazzaferro V, Hilgard P, Gane E, Blanc J-F, et al. Sorafenib in advanced hepatocellular carcinoma. N Engl J Med. 2008 Jul 24;359(4):378-90.

6 Cheng AL, Kang YK, Chen Z, Tsao CJ, Qin S, Kim JS, et al. Efficacy and safety of sorafenib in patients in the Asia-Pacific region with advanced hepatocellular carcinoma: a phase III randomised, double-blind, placebo-controlled trial. Lancet Oncol. 2009 Jan;10(1):2534.

7 Kudo M, Finn RS, Qin S, Han K-H, Ikeda K, Piscaglia F, et al. Lenvatinib versus sorafenib in first-line treatment of patients with unresectable hepatocellular carcinoma: a randomised phase 3 non-inferiority trial. Lancet. 2018 Mar 24;391(10126):1163-73.

8 Bruix J, Qin S, Merle P, Granito A, Huang YH, Bodoky G, et al. Regorafenib for patients with hepatocellular carcinoma who progressed on sorafenib treatment (RESORCE): a randomised, double-blind, placebo-controlled, phase 3 trial. Lancet. 2017 Jan 7;389(10064): 56-66.

9 Zhu AX, Kang Y-K, Yen C-J, Finn RS, Galle PR, Llovet JM, et al. Ramucirumab after sorafenib in patients with advanced hepatocellular carcinoma and increased $a$-fetoprotein concentrations (REACH-2): a randomised, double-blind, placebo-controlled, phase 3 trial. Lancet Oncol. 2019 Feb;20(2):282-96.

10 Abou-Alfa GK, Meyer T, Cheng A-L, ElKhoueiry AB, Rimassa L, Ryoo B-Y, et al. Cabozantinib in patients with advanced and progressing hepatocellular carcinoma. N Engl J Med. 2018 Jul 5;379(1):54-63.

11 El-Khoueiry AB, Sangro B, Yau T, Crocenzi TS, Kudo M, Hsu C, et al. Nivolumab in patients with advanced hepatocellular carcinoma (CheckMate 040): an open-label, noncomparative, phase $1 / 2$ dose escalation and expansion trial. Lancet. 2017 Jun 24; 389(10088):2492-502.

12 Zhu AX, Finn RS, Edeline J, Cattan S, Ogasawara $\mathrm{S}$, Palmer D, et al. Pembrolizumab in patients with advanced hepatocellular carcinoma previously treated with sorafenib (KEYNOTE-224): a non-randomised, open-label phase 2 trial. Lancet Oncol. 2018 Jul;19(7):940-52.

13 Kudo M. Immuno-oncology therapy for hepatocellular carcinoma: current status and ongoing trials. Liver Cancer. 2019;8(4):221-38.

14 Finn RS, Qin S, Ikeda M, Galle PR, Ducreux M, Kim T-Y, et al. Atezolizumab plus bevacizumab in unresectable hepatocellular carcinoma. N Engl J Med. 2020 May 14;382(20): 1894-905.

15 Yau T, Park JW, Finn RS, Cheng A-L, Mathurin P, Edeline J, et al. LBA38_PR:
CheckMate 459: a randomized, multi-center phase 3 study of nivolumab (NIVO) vs sorafenib (SOR) as first-line (1L) treatment in patients (pts) with advanced hepatocellular carcinoma (aHCC). ESMO2019. Ann Oncol. 2019;30(Suppl 5):v874-5.

16 Finn RS, Ryoo B-Y, Merle P, Kudo M, Bouattour M, Lim HY, et al. Pembrolizumab as second-line therapy in patients with advanced hepatocellular carcinoma in KEYNOTE-240: a randomized, double-blind, phase III trial. J Clin Oncol. 2020 Jan 20;38(3):193-202.

17 Sia D, Jiao Y, Martinez-Quetglas I, Kuchuk O, Villacorta-Martin C, Castro de Moura M, et al. Identification of an immune-specific class of hepatocellular carcinoma, based on molecular features. Gastroenterology. 2017 Sep;153(3): $812-26$.

18 Pinyol R, Sia D, Llovet JM. Immune exclusionWnt/CTNNB1 class predicts resistance to immunotherapies in HCC. Clin Cancer Res. 2019 Apr 1;25(7):2021-3.

19 Llovet JM, Montal R, Sia D, Finn RS. Molecular therapies and precision medicine for hepatocellular carcinoma. Nat Rev Clin Oncol. 2018 Oct;15(10):599-616.

20 Harding JJ, Nandakumar S, Armenia J, Khalil DN, Albano M, Ly M, et al. Prospective genotyping of hepatocellular carcinoma: clinical implications of next-generation sequencing for matching patients to targeted and immune therapies. Clin Cancer Res. 2019 Apr 1;25(7): 2116-26.

21 Morita M, Nishida N, Sakai K, Aoki T, Chishina $\mathrm{H}$, Takita $\mathrm{M}$, et al. Immunological microenvironment predicts the survival of the patients with hepatocellular carcinoma treated with anti-PD-1 antibody. Liver Cancer. Forthcoming 2021. 
22 Ichikawa T, Saito K, Yoshioka N, Tanimoto A, Gokan T, Takehara Y, et al. Detection and characterization of focal liver lesions: a Japanese phase III, multicenter comparison between gadoxetic acid disodium-enhanced magnetic resonance imaging and contrast-enhanced computed tomography predominantly in patients with hepatocellular carcinoma and chronic liver disease. Invest Radiol. 2010 Mar;45(3):133-41.

23 Ahn SS, Kim MJ, Lim JS, Hong HS, Chung YE, Choi JY. Added value of gadoxetic acid-enhanced hepatobiliary phase MR imaging in the diagnosis of hepatocellular carcinoma. Radiology. 2010 May;255(2):459-66.

24 Narita M, Hatano E, Arizono S, MiyagawaHayashino A, Isoda H, Kitamura K, et al. Expression of OATP1B3 determines uptake of Gd-EOB-DTPA in hepatocellular carcinoma. J Gastroenterol. 2009;44(7):793-8.

25 Kitao A, Zen Y, Matsui O, Gabata T, Kobayashi S, Koda W, et al. Hepatocellular carcinoma: signal intensity at gadoxetic acid-enhanced MR Imaging: correlation with molecular transporters and histopathologic features. Radiology. 2010 Sep;256(3):817-26.

26 Kitao A, Matsui O, Yoneda N, Kozaka K, Shinmura R, Koda W, et al. The uptake transporter OATP8 expression decreases during multistep hepatocarcinogenesis: correlation with gadoxetic acid enhanced MR imaging. Eur Radiol. 2011 Oct;21(10):2056-66.

27 Kitao A, Matsui O, Yoneda N, Kozaka K, Kobayashi S, Koda W, et al. Hypervascular hepatocellular carcinoma: correlation between biologic features and signal intensity on gadoxetic acid-enhanced MR images. Radiology. 2012 Dec;265(3):780-9.

28 Ueno A, Masugi Y, Yamazaki K, Komuta M, Effendi K, Tanami $Y$, et al. OATP1B3 expression is strongly associated with $\mathrm{Wnt} / \beta$-catenin signalling and represents the transporter of gadoxetic acid in hepatocellular carcinoma. J Hepatol. 2014 Nov;61(5):1080-7.

29 Kitao A, Matsui O, Yoneda N, Kozaka K, Kobayashi S, Sanada J, et al. Hepatocellular carcinoma with beta-catenin mutation: imaging and pathologic characteristics. Radiology. 2015 Jun;275(3):708-17.

30 Kudo M. Gd-EOB-DTPA-MRI could predict $\mathrm{WNT} / \beta$-catenin mutation and resistance to immune checkpoint inhibitor therapy in hepatocellular carcinoma. Liver Cancer. 2020 Sep;9(5):479-90.

31 Marrero JA, Kulik LM, Sirlin CB, Zhu AX, Finn RS, Abecassis MM, et al. Diagnosis, staging, and management of hepatocellular carcinoma: 2018 practice guidance by the American Association for the Study of Liver Diseases. Hepatology. 2018 Aug;68(2):723-50.

32 Oken MM, Creech RH, Tormey DC, Horton J, Davis TE, McFadden ET, et al. Toxicity and response criteria of the Eastern Cooperative Oncology Group. Am J Clin Oncol. 1982 Dec; 5(6):649-55.
33 Trotti A, Byhardt R, Stetz J, Gwede C, Corn B, Fu K, et al. Common toxicity criteria: version 2.0. an improved reference for grading the acute effects of cancer treatment: impact on radiotherapy. Int J Radiat Oncol Biol Phys. 2000 Apr 1;47(1):13-47.

34 Liu YJ, Zhu GP, Guan XY. Comparison of the NCI-CTCAE version 4.0 and version 3.0 in assessing chemoradiation-induced oral mucositis for locally advanced nasopharyngeal carcinoma. Oral Oncol. 2012 Jun;48(6):554-9.

35 Therasse P, Arbuck SG, Eisenhauer EA, Wanders J, Kaplan RS, Rubinstein L, et al. New guidelines to evaluate the response to treatment in solid tumors. European Organization for Research and Treatment of Cancer, National Cancer Institute of the United States, National Cancer Institute of Canada. J Natl Cancer Inst. 2000 Feb 2;92(3):205-16.

36 Development. NCIP. Cancer therapy evaluation program. Available from: https://ctepcancergov/protocolDevelopment/electronic_ applications/ctchtm. Accessed 2017 Mar 21.

37 Tsuboyama T, Onishi H, Kim T, Akita H, Hori M, Tatsumi M, et al. Hepatocellular carcinoma: hepatocyte-selective enhancement at gadoxetic acid-enhanced MR imaging: correlation with expression of sinusoidal and canalicular transporters and bile accumulation. Radiology. 2010 Jun;255(3):824-33.

38 Sterling RK, Lissen E, Clumeck N, Sola R, Correa MC, Montaner J, et al. Development of a simple noninvasive index to predict significant fibrosis in patients with HIV/HCV coinfection. Hepatology. 2006 Jun;43(6):1317-25.

39 US Food \& Drug Administration. Nivolumab (Opdivo). 2014 [updated 2019 May 2]. Available from: https://www.accessdata.fda.gov/ scripts/cder/daf/index.cfm? event=overview. process\&ApplNo $=125554$.

40 US Food \& Drug Administration. Pembrolizumab (Keytruda). 2014 [updated 2019 Apr 19]. Available from: http://www.accessdata.fda.gov/ scripts/cder/daf/index.cfm? event=overview. process\&varApplNo=125514.

41 US Food \& Drug Administration. Atezolizumab (Tecentriq). 2016 [updated 2019 May 6]. Available from: https://www.accessdata. $\mathrm{fda} \cdot \mathrm{gov} / \mathrm{scripts} / \mathrm{cder} / \mathrm{d}$ af/index. $\mathrm{cfm}$ ? event=overview.process\& Ap$\mathrm{plNo}=761034$.

42 US Food \& Drug Administration. Ipilimumab (Yervoy). 2011 [updated 2019 May 8]. Available from: https://clinicaltrials.gov/ct2/show/ NCT03695952? term = pembrolizumab \& cond=Hepatobiliary+Cancer\&rank $=1$.

43 Xie QK, Zhao YJ, Pan T, Lyu N, Mu LW, Li SL, et al. Programmed death ligand 1 as an indicator of pre-existing adaptive immune responses in human hepatocellular carcinoma. Oncoimmunology. 2016 Jul;5(7):e1181252.

44 Nishida N, Sakai K, Morita M, Aoki T, Takita M, Hagiwara S, et al. Association between genetic and immunological background of hepatocellular carcinoma and expression of pro- grammed cell death-1. Liver Cancer. 2020 Aug;9(4):426-39.

45 Torbenson MS, Ng IO, Park YN, Roncalli M, Sakamoto M. In: WHO Classification of Tumours Editorial Board, editor. Digestive system tumours: WHO classification of tumours. 5th ed. Geneva: WHO Press; 2019. p. 229-39.

46 Chabot V, Reverdiau P, Iochmann S, Rico A, Sénécal D, Goupille C, et al. CCL5-enhanced human immature dendritic cell migration through the basement membrane in vitro depends on matrix metalloproteinase-9. J Leukoc Biol. 2006 Apr;79(4):767-78.

47 Ruiz de Galarreta M, Bresnahan E, MolinaSánchez P, Lindblad KE, Maier B, Sia D, et al. $\beta$-Catenin activation promotes immune escape and resistance to anti-PD-1 therapy in hepatocellular carcinoma. Cancer Discov. 2019 Aug;9(8):1124-41.

48 Paterson AC, Sciot R, Kew MC, Callea F, Dusheiko GM, Desmet VJ. HLA expression in human hepatocellular carcinoma. Br J Cancer. 1988 Apr;57(4):369-73.

49 Berko D, Carmi Y, Cafri G, Ben-Zaken S, Sheikhet HM, Tzehoval E, et al. Membraneanchored $\beta 2$-microglobulin stabilizes a highly receptive state of MHC class I molecules. J Immunol. 2005 Feb 15;174(4):2116-23.

50 Mittal D, Gubin MM, Schreiber RD, Smyth MJ. New insights into cancer immunoediting and its three component phases: elimination, equilibrium and escape. Curr Opin Immunol. 2014 Apr;27:16-25.

51 Garcia-Diaz A, Shin DS, Moreno BH, Saco J, Escuin-Ordinas H, Rodriguez GA, et al. Interferon receptor signaling pathways regulating PD-L1 and PD-L2 expression. Cell Rep. 2017 May 9;19(6):1189-201.

52 Kan Z, Zheng H, Liu X, Li S, Barber TD, Gong $Z$, et al. Whole-genome sequencing identifies recurrent mutations in hepatocellular carcinoma. Genome Res. 2013 Sep;23(9):1422-33.

53 Tsujikawa H, Masugi Y, Yamazaki K, Itano O, Kitagawa Y, Sakamoto M. Immunohistochemical molecular analysis indicates hepatocellular carcinoma subgroups that reflect tumor aggressiveness. Hum Pathol. 2016 Apr;50:24-33.

54 Yamashita T, Kitao A, Matsui O, Hayashi T, Nio K, Kondo M, et al. Gd-EOB-DTPA-enhanced magnetic resonance imaging and alpha-fetoprotein predict prognosis of earlystage hepatocellular carcinoma. Hepatology. 2014 Nov;60(5):1674-85.

55 Yamauchi M, Ono A, Ishikawa A, Kodama K, Uchikawa S, Hatooka H, et al. Tumor fibroblast growth factor receptor 4 level predicts the efficacy of lenvatinib in patients with advanced hepatocellular carcinoma. Clin Transl Gastroenterol. 2020 May;11(5):e00179.

56 Aoki T, Kudo M, Ueshima K, Morita M, Chishina H, Takita M, et al. Exploratory analysis of lenvatinib therapy in patients with unresectable hepatocellular carcinoma who have failed prior PD-1/PD-L1 checkpoint blockade. Cancers. 2020 Oct 20;12(10):3048. 